\title{
Emerging deep learning techniques using magnetic resonance imaging data applied in multiple sclerosis and clinical isolated syndrome patients (Review)
}

\author{
ELEFTHERIOS E. KONTOPODIS ${ }^{1,2}$, EFROSINI PAPADAKI ${ }^{1,2}$, ELEFTHERIOS TRIVIZAKIS ${ }^{1,2}$, \\ THOMAS G. MARIS ${ }^{1,2}$, PANAGIOTIS SIMOS ${ }^{1,3}$, GEORGIOS Z. PAPADAKIS ${ }^{1,2}$, ARISTIDIS TSATSAKIS ${ }^{4}$, \\ DEMETRIOS A. SPANDIDOS ${ }^{5}$, APOSTOLOS KARANTANAS ${ }^{1,2}$ and KOSTAS MARIAS ${ }^{1,6}$ \\ ${ }^{1}$ Computational BioMedicine Laboratory, Institute of Computer Science, Foundation for Research and \\ Technology-Hellas; Departments of ${ }^{2}$ Radiology and ${ }^{3}$ Psychiatry and Behavioral Sciences, Medical School, \\ University of Crete, 70013 Heraklion; ${ }^{4}$ Centre of Toxicology Science and Research, Faculty of Medicine; \\ ${ }^{5}$ Laboratory of Clinical Virology, Medical School, University of Crete, 71003 Heraklion; ${ }^{6}$ Department of \\ Electrical and Computer Engineering, Hellenic Mediterranean University, 71410 Heraklion, Greece
}

Received April 12, 2021; Accepted July 29, 2021

DOI: $10.3892 /$ etm.2021.10583

\begin{abstract}
Computer-aided diagnosis systems aim to assist clinicians in the early identification of abnormal signs in order to optimize the interpretation of medical images and increase diagnostic precision. Multiple sclerosis (MS) and clinically isolated syndrome (CIS) are chronic inflammatory, demyelinating diseases affecting the central nervous system. Recent advances in deep learning (DL) techniques have led to novel computational paradigms in MS and CIS imaging designed for automatic segmentation and detection of areas of interest and automatic classification of anatomic structures, as well as
\end{abstract}

Correspondence to: Dr Eleftherios E. Kontopodis, Computational BioMedicine Laboratory, Institute of Computer Science, Foundation for Research and Technology-Hellas, 100 Nikolaou Plastira Street, Vassilika Vouton, 70013 Heraklion, Greece

E-mail: 1kontopodis@gmail.com

Abbreviations: MS, multiple sclerosis; CNS, central nervous system; BBB, blood brain barrier; CIS, clinically isolated syndrome; MRI, magnetic resonance imaging; CA, contrast agent; FLAIR, fluid-attenuated inversion recovery; CSF, cerebrospinal fluid; DWI, diffusion weighted imaging; AI, artificial intelligence; CAD, computer aided diagnosis; DL, deep learning; GM, gray matter; CNNs, convolution neural networks; NAWM, normal appearing white matter; NAGM, normal appearing gray matter; TPR, true positive rate; FPR, false positive rate; FCNN, fully convolutional neural network; AUC, area under curve; DBN, deep belief network; HC, healthy controls; PReLU, parametric rectified linear unit; LRP, layer-wise relevance propagation; NMOSD, neuromyelitis optical spectrum disorder; EDSS, expanded disability status scale; PET, positron emission tomography; MWF, myelin water fraction

Key words: magnetic resonance imaging/diagnosis, multiple sclerosis, deep learning, clinical isolated syndrome optimization of neuroimaging protocols. To this end, there are several publications presenting artificial intelligence-based predictive models aiming to increase diagnostic accuracy and to facilitate optimal clinical management in patients diagnosed with MS and/or CIS. The current study presents a thorough review covering DL techniques that have been applied in MS and CIS during recent years, shedding light on their current advances and limitations.

\section{Contents}

1. Introduction

2. Selection criteria

3. Detection: Segmentation methods

4. Classification: Diagnosis

5. Post processing techniques and image enhancement methods

6. Discussion

\section{Introduction}

Multiple Sclerosis (MS) is a chronic inflammatory demyelinating disease of the central nervous system (CNS), commonly affecting young adults. MS usually manifests as a relapsing-remitting (RR) process, predominantly characterized by inflammatory demyelination that secondarily evolves to a progressive stage with neurodegeneration, gliosis and accumulating disability. Although the etiology of MS is largely unknown, it is considered primarily an autoimmune disease, in which activated myelin-specific T-cells, migrate from the periphery to the CNS, by crossing the blood brain barrier (BBB) inducing the formation of new inflammatory demyelinating lesions $(1,2)$. Clinically isolated syndrome (CIS) describes a clinical episode of at least $24 \mathrm{~h}$, suggestive of an inflammatory demyelinating disorder of the CNS. CIS usually 
occurs in young adults and represents a monophasic episode, isolated in time, and usually isolated in space, affecting optic nerves, the brainstem, or the spinal cord, while there are no signs of fever or infection. Although patients usually recover from their presenting episode, CIS is often the first manifestation of MS. The course of MS after CIS is variable: after 15-20 years, one third of patients have a benign course with minimal or no disability, while half will have developed secondary progressive MS with increasing disability $(3,4)$. MS and CIS clinical studies, need to be accompanied by sensitive and reliable imaging methods, in order to investigate the specific pathological alterations of the white matter (WM). Imaging methods for MS include optical computed tomography and coherent anti-Stokes Raman scattering microscopy (5). Magnetic Resonance Imaging (MRI) is the modality of choice for diagnosis and monitoring MS pathology, by exploiting both conventional and quantitative protocols (6).

Conventional MR techniques for MS and CIS imaging, include T1-w images since in this protocol lesions are usually depicted by lower signal intensity compared to the surrounding tissues, while T1 severe hypointense lesions (black holes) represent the most severe stage of MS lesions with irreversible axonal loss $(5,7)$. Lesions in earlier stages of the disease can be captured using Gd enhanced T1-w MRI sequences, based on the fact that contrast agent (CA) uptake indicates premature stage of inflammation and blood brain barrier (BBB) disruption. Furthermore, T2-w MRI sequences are also sensitive in detecting lesions, since these are demonstrated as bright areas in a dark background. T2 hyperintense lesions represent tissues that are characterized by edema, inflammation, demyelination, axonal loss and gliosis. Sensitivity is further enhanced by using the fluid-attenuated inversion recovery (FLAIR) protocol, which can be used for highlighting subcortical and periventricular lesions by suppressing T2 signal from cerebrospinal fluid (CSF) (8). The aforementioned techniques constitute the gold standard for MS and CIS diagnosis and monitoring (9).

However, conventional MRI do not provide sufficient sensitivity that could enable early diagnosis or appropriate specificity to predict disease severity. Quantitative MRI techniques provide enhanced insights in disease severity and tissue damage. These techniques include: i) MR spectroscopy which provides a non-invasive method to examine the biochemical changes in MS (10); ii) magnetization transfer imaging which offers improved sensitivity and specificity for MS studies (11,12); iii) diffusion weighted imaging (DWI) and diffusion tensor imaging (DTI) which are quantitative MRI techniques, providing information on size, integrity, geometry, and orientation of tissue fibres by capturing the motion of tissue water (13); iv) dynamic contrast enhanced MRI which enables quantification of BBB disruption, a therapeutic target in MS (14); and v) dynamic susceptibility contrast MRI that provides quantitative maps of cerebral blood flow, cerebral blood volume and temporal parameters such as mean transit time by intravenous administration of CA (14).

Conventional clinical protocols for MS diagnosis and monitoring, utilize imaging data and measurements, such as signal intensities and volumetric results from user defined regions of interest, in order to compare healthy and MS subjects or to examine longitudinal changes during therapy, and evaluate the clinical outcome. However, these results are prone to errors due to inter-observer variability, while the lack of ability to compare studies from different modalities is a major limitation. Moreover, procedures that involve human interplay, besides that are time consuming and employ human experts, these may be characterized by high inter observer variability that may hinder the quality of the final results. Therefore, during the last years artificial intelligence (AI) techniques have opened new horizons in computer aided diagnosis (CAD) systems, by automatically generalizing rules and patterns that exist in labeled imaging data, while by utilizing this information they are able to generate predictions and classifications on independent datasets that were not used in the model training process (15).

Furthermore, segmentation techniques in MS imaging are gaining ground during the last years, considering the necessity of accurate algorithms for automatic delineation of anatomical structures (16). Performing these tasks manually is time-consuming and prone to errors, thus there is a lot of interest to accomplish this task using automated computer algorithms, towards increased accuracy and precision, while at the same time minimize human involvement.

Finally, computer algorithms able to assess image quality of the acquired data, as well as methods that are able to compute optimal protocol parameters prior to image acquisition, are tools that can save time and provide noise free and qualitative data with increased diagnostic information (17). To this purpose, AI techniques constitute a valuable methodology for assessing MR image quality as well as determining the necessity to repeat the acquisition, while there are attempts to provide algorithms aiming to image protocol optimization.

The focus of this review paper, is to study the current literature regarding MR imaging deep learning (DL) applications focused on MS and CIS imaging. The reviewed publications were examined from different standpoints including the different DL architectures, the patient cohorts and the end-point of the reported studies in the field.

\section{Selection criteria}

Based on a PubMed search using keywords: 'deep learning' AND 'multiple sclerosis', 74 articles were initially identified. Subsequently, screening for MR imaging relevance and removing duplicates led to 32 original research articles that applied DL techniques in MS and CIS for diagnosis tasks, segmentation tasks and clinical protocol optimization. Afterwards, a similar search in google scholar, using the same keywords, and keeping only original research journal papers, led to 13 additional articles making a total of 45 original papers.

After an initial review, selected articles were separated in three broad categories i.e., i) segmentation of MS and CIS lesions; ii) classification of different pathologies and anatomical structures; and iii) post processing techniques and image enhancement methods with application in MS and CIS imaging. In Fig. 1 presents the general workflow of the DL techniques along with the final goal, as was determined in the present review manuscript. In Fig. 2, the distribution of the reviewed papers in each of the three abovementioned categories is presented. Obviously, segmentation and classification techniques are the main areas of interest, accounting for the 

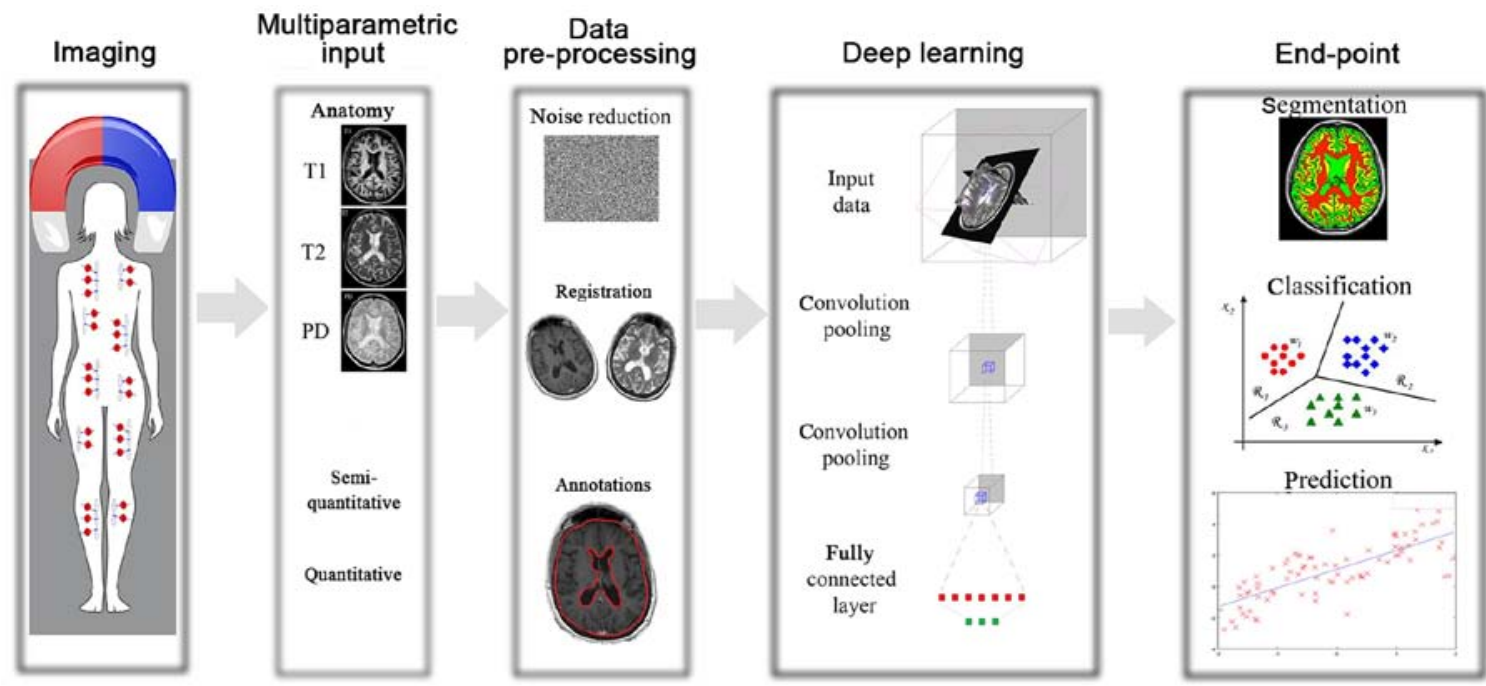

Figure 1. A schematic generalized workflow of the DL techniques presented in the manuscript. Initially the magnetic resonance acquisition is performed, producing a number of different anatomical and functional map representations. These provide additional information regarding the underlying pathophysiology (for example, quantitative), which can be used as input in the DL architecture for training the models and, in turn, address un-met clinical needs. DL, deep learning.

Distribution of publications through categories

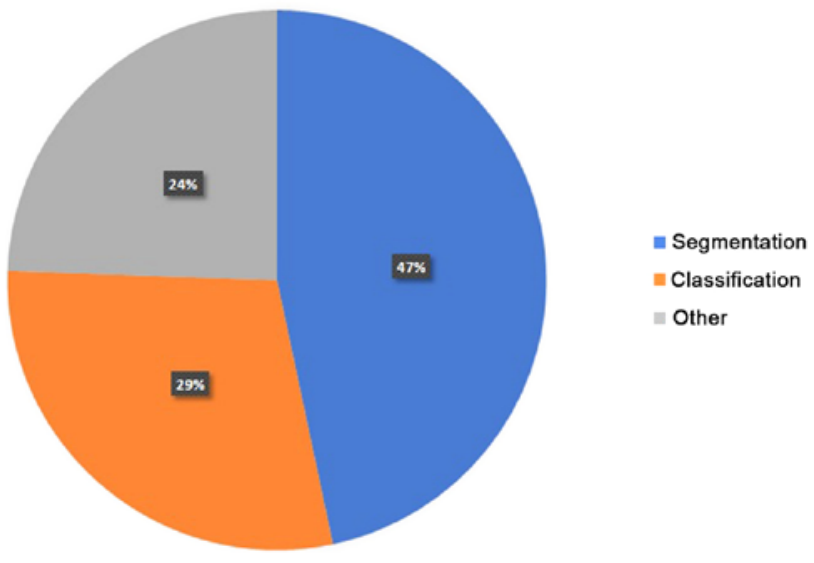

Figure 2. Pie chart distribution, grouping reviewed studies according to different end-points. The vast majority of published articles are serving segmentation and classification techniques.

$76 \%$ of the overall publications. Moreover, in Fig. 3 the number of publications through years, for the three abovementioned application fields, is presented.

\section{Detection: Segmentation methods}

MS is a progressive disease of CNS affecting the myelin sheath, a fatty material that envelops neuron axons, and as a result alters the morphology and structure of the brain. Furthermore, brain atrophy is taking place in cortical and subcortical regions, whereas the gray matter (GM) atrophy and its association with disability and cognitive impairment is under investigation $(18,19)$. Nevertheless, measurement of GM volume is a useful method for assessing the overall GM damage and can be estimated through conventional MRI techniques (6). Another established measurement for prediction of neurological disorders is the lesion load (20), since it has been proved that both lesion volume and lesion count are strong predictors of disease course and progressive timepoints. Additionally, detection of MS lesion is of paramount importance since the diagnosis of MS is based on the spatial and temporal distribution of focal demyelination lesions (21). Since manual detection of MS lesions is time consuming and prone to errors as well as inter observer variability, algorithms aiming to automatically delineate these tissues may constitute a great tool for clinical practice. Considering all the aforementioned, automated computer algorithms aiming to segment anatomical structures as well as to detect MS lesions, are valuable tools for clinicians offering objective and repeatable results. Accurate segmentation of WM lesions is hindered due to overlapping intensities of these lesions with GM, while finite resolution of MR images, complicated shapes that vary among different lesions as well as the partial volume effect, hamper the accurate delineation of MS tissues. Additionally, gray matter lesions are often not visible in T2-w images due to size, magnetic relaxation characteristics and partial volume effects with CSF. Finally, these lesions may not be visible even in FLAIR sequences, despite the increased sensitivity of this protocol compared to a conventional T2-w one.

Many previous studies have proposed DL methods aiming to provide a robust and reliable framework for detection and segmentation of MS lesions, while the most significant of are summarized below. Valverde et al (22) presented an automated WM lesions segmentation method, by utilizing a cascade of two 3D patch-wise convolution neural networks $(\mathrm{CNN})$, the first was sensitive for revealing possible candidate lesions voxels while the second one was responsible for reducing the number of misclassified voxels resulted from the first network. The accuracy of the proposed approach was evaluated in the medical image computing and computer assisted intervention society (MICCAI) 2008 database (http://www.ia.unc. edu/MSseg), as well as on two private MS clinical datasets, while results were also compared with other lesion segmentation tools and methods. It was reported that the proposed 
Temporal distibution of publications

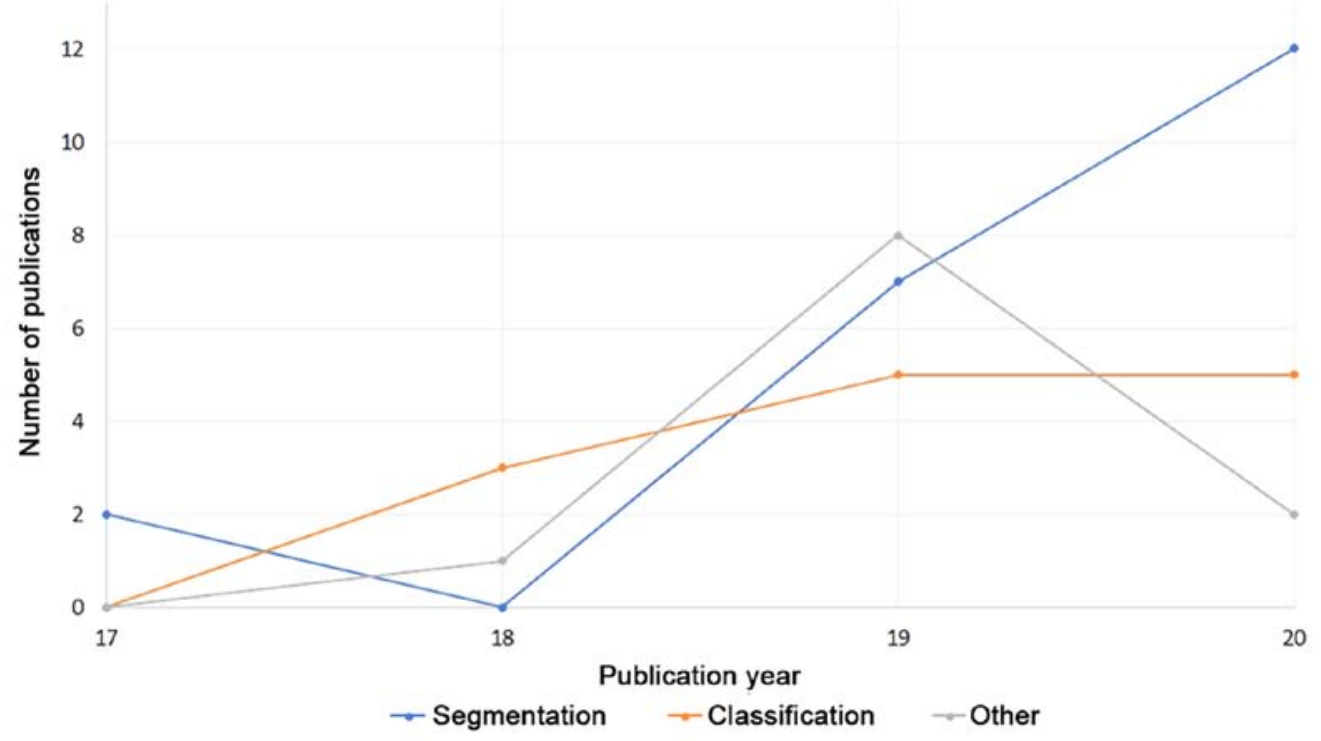

Figure 3. Number of publications, in each application category, per year. There is a trend indicating that the number of publications in the segmentation and classification categories are increasing in recent years.

approach is the best ranked on the MICCAI2008 challenge, and in the clinical datasets it exhibited an increased accuracy in the WM lesions segmentations.

Birendaum et al (23) developed an automated DL method for MS lesion segmentation, utilizing a CNN that was mainly based on the Single View CNN (V-Net) and the Longitudinal Network (L-Net). For this work, the 2015 Longitudinal MS Lesion Segmentation Challenge dataset (24) was used while the network resulted in Dice Similarity Coefficient 0.627. Finally, authors reported that the aforementioned method achieved performance level comparable to a trained human rater.

Gros et al (25), proposed a fully-automated framework for segmenting spinal cord and intramedullary MS lesions using conventional MRI data. The aim of this study was to provide a robust and automated framework, by overcoming the technical limitations of large variabilities related to acquisition parameters and image artifacts, and furthermore to eliminate the inter-rater variability as well as to optimize the large-throughput analysis pipeline. The cohort of this study consisted of 1,042 subjects; 459 healthy controls, $471 \mathrm{MS}$ patients and 112 with other spinal pathologies. Authors used a sequence of two CNNs, the first intended for the detection of spinal cord centerline using 2D convolutions, while the second segmented the spinal cord and/or lesions using 3D convolutions. These networks were trained independently using the Dice loss (26), considering that it is not sensitive to high class imbalances, while comparing the proposed technique with the PropSeg method, an unsupervised technique for spinal cord segmentation (27), resulted in median Dice 95\% when using manual vs $88 \%$ when using PropSeg. Moreover, regarding MS lesion segmentation, the proposed technique provided Dice of $60 \%$, while sensitivity and precision were $83 \%$ and $77 \%$ respectively.

Aslani et al (28) presented an automated approach for MS lesion segmentation, based on a Deep end-to-end 2D CNN, a technique that incorporates a multi-branch down-sampling path which enables the network to encode information stemming from multiple modalities in a separate way. The proposed model was trained and tested by using orthogonal plane orientations for every 3D modality, in order to exploit information from all directions. Evaluation of the proposed method was on two datasets, one private (37 MS patients) as well as the International Symposium on Biomedical Imaging (ISBI) 2015 longitudinal MS lesion segmentation challenge dataset (14 MS patients) (24). Results on the ISBI challenge ranked the proposed methodology among the top (DSC 0.6114), while on the private dataset the proposed method achieved the best results (DSC 0.6655).

Sander et al (29), employed a fully automated segmentation method, based on multi-dimensional gated recurrent units (MD-GRU). MD-GRU, is a DL approach that employs a convolutional adaptation of a recurrent neural architecture. This network was trained on 50 MS patients and 17 healthy controls, while a refinement procedure of the algorithm parameters took place in a cohort of 20 independent MS patients. For accuracy testing, 20 MS or CIS patients from the same cohort were used, while accuracy was further assessed in a dataset including 80 independent MS or CIS patients from the same cohort. Reproducibility assessment was followed by considering T1-w images from 33 healthy controls. Finally, robustness of the segmentation approach was assessed using images from 50 Alzheimer patients. According to the results, the proposed segmentation approach provided accurate, highly reproducible and a robust fully-automated segmentation framework for the brainstem and its substructure in both healthy controls as well as in MS and Alzheimer disease patients. In more detail, study results were compared with FreeSurfer (30) software results, while authors reported that the mean $\%$ change/SD between test-retest brainstem volumes were $0.45 \% / 0.005$ for MD-GRU and 0.95\%/0.009 for FreeSurfer and 0.86\%/0.007 for manual segmentations. 
MD-GRU segmentations were compared to segmentations performed from experts, resulted in mean Dice score/SD of 0.97/0.005 for brainstem, 0.95/0.0131 for mesencephalon, $0.98 / 0.006$ for pons and $0.95 / 0.015$ for medulla oblongata.

Hashemi et al (31), developed a 3D fully connected convolution neural network (FCNN) with DenseNet blocks (FC-DenseNet), using an asymmetric similarity loss function Tversky index, aiming to mitigate the data imbalance problem and to achieve a better trade-off among precision and recall. This method used 15 patients from the MS lesion segmentation challenge (MSSEG) of the 2016 Medical Image Computing and Computer Assisted Intervention conference (32), as well as 5 patients from the longitudinal MS lesion segmentation challenge of the IEEE International Symposium on Biomedical Imaging (ISBI) conference (33). For training purposes, a 5 -fold cross validation strategy was utilized, in which five instances of the training were performed on 4/5th of each dataset and validated on the remaining $1 / 5$ th. For testing the implemented architecture, due to limited number of subjects 5 -fold cross validation was used. Study results report that MS lesion segmentation presented improved performance evaluation metrics by using asymmetry similarity loss function rather than using the Dice similarity in the loss layer. Furthermore, authors reported that the proposed framework achieved improved precision-recall trade-off and average DSC scores of $69.9 \%$ and $65.74 \%$ for MSSEG 2016 and ISBI longitudinal MS lesion segmentation challenge correspondingly.

Gabr et al (34) investigated the performance of FCNN in segmenting brain tissues using a larger cohort of MS patients. The MRI protocols and the patient cohort used in this study, included a dual echo fast spin echo (FSE) sequence, a T1-w sequence and FLAIR sequence from a cohort of 1,000 RRMS patients emanating from the CombiRx clinical trial, while training, validation and testing of the FCNN were based on the aforementioned data. Moreover, automated segmentation results were validated by two experts, while for cross-validation of the results, the leave-one-centre-out approach was used. Results from this study, report high average Dice similarity coefficient for all segmented tissues ( 0.95 for WM, 0.96 for GM, 0.82 for CSF and 0.82 for T2 lesions), and high correlations among the DL segmented tissues and the annotations from the expert $(\mathrm{R} 2>0.92)$. Finally, cross validation reported consistency of the results among the different centers.

Weeda et al (35) compared four different lesion segmentation methods to investigate the suitability of the DL CNN method nicMSlesions (36), in an independent dataset aiming to determine whether this method is suitable for larger, multi-center studies. For this purpose, manual segmentation was performed in 14 MS subjects, while five different automated lesion segmentation methods were compared: i) unsupervised-untrained LesionTOADS (37); ii) supervised-untrained methods with threshold adjustment LST-LPA (38) and nicMSlesions with default settings; iii) supervised-untrained LST-LPA with threshold adjustment; iv) supervised-trained with leave-one-out cross-validation nicMSlesions and BIANCA (39); and v) supervised-trained on a single subject nicMSlesions. Results from this study report that the best volumetric and spatial agreement with manual annotations was obtained with the supervised and trained methods nicMSlesions and BIANCA, while the worst results was provided by the unsupervised, untrained method lesionTOADS, concluding that input from a single subject to fine tune a DL CNN is sufficient for lesion segmentation.

McKinley et al (40) presented a segmentation framework based on a cascade of two CNNs, providing multimodal MR images segmentation into lesion, normal appearing white matter (NAWM) and normal appearing gray matter (NAGM) structures. For this purpose, MRI exams from 122 RRMS patients were used ( 90 patients for training and validation of the classifier, 32 patients for testing), while a second dataset including $10 \mathrm{MRI}$ datasets was used for direct comparison with other centers. Results in the current study reported that both Dice coefficient as well as specificity and sensitivity are improved compared to previous approaches, while a good agreement with individual human raters was achieved. Moreover, authors concluded that the proposed method performed well on data emanating from different centers, scanners and field-strengths that were not represented in the training dataset indicating good generalizability, while it was also reported that the classifier achieved to identify lesions missed by human raters.

Narayana et al (41) studied the dependence of DL segmentation accuracy on the training size, aiming to define the required training set for an adequate accuracy in brain MRI segmentation in MS patients. For this purpose, a 2D FCNN was trained using 16 different training sizes, while segmentation accuracy was determined according to the training size, and network performance was evaluated by the dice similarity coefficient and lesion true-positive and false positive-rates. The presented methodology was evaluated in MRI data from a cohort of 1,008 MS patients, while the automatic segmentation results were assessed by two neuroimaging experts. Study results report that lesion segmentation showed stronger dependency to the sample size comparing with the GM, WM and CSF, concluding that excellent results were provided by a training set of 10 image volumes for GM, WM and CSF. Finally training size of at least 50 images was necessary for adequate lesion segmentation with DSC $<0.68$ while for 150 sample size the DSC was 0.82 .

Nair et al (42) presented a 3D MS lesion detection and segmentation CNN. The network was trained using a large scale, multi-site, multi-scanner clinical MS dataset consisted of 1,064 RRMS patients scanned annually over a period of 24 months, while imaging protocol included T2w, T1-w, FLAIR and proton density (PD) images. The network was trained using a weighted binary cross-entropy loss, while the performance of the network was evaluated separately in i) a voxel-level analysis; and ii) lesion-level analysis. Study results report that uncertainty filtering vastly improves lesion detection accuracy for small lesions.

In the same dataset, McKinley et al (43) trained two state-of-the-art CNNs architectures, a 3D Unet consisting a reference implementation and a more recently proposed architecture, the DeepSCAN, in order to segment multimodal MR images into lesion classes and NAWM and NAGM structures. Results from this study reported that both examined methods outperformed previous approaches in the literature related to the MSSEG dataset, while a good agreement among the automated segmentations and results from human ratters is reported. Moreover, DeepSCAN network found to achieve 
the best performance in both lesion and anatomical labelling, while there is reported that this classifier recognized lesions that were missed by human ratters.

Narayana et al (44) investigated the effects that different combinations of multi-contrast MR images may infer when serving as input to a CNN. To this end, U-net, a fully CNN was used in order to automatically segment GM, WM, CSF and lesions in 1,000 MS patients. Image datasets included T1-w images, FLAIR and dual echo turbo spin echo or fast spin echo images. For the assessment of the segmentation performance, the DSC was evaluated, while for lesions the true positive rate (TPR) and false positive rate (FPR) were also reported. Results from this study report that when segmentation input was a combination of all four image modality data, it was achieved the highest DSC for all tissue volumes, while high DSC results were also obtained when FLAIR protocol was included in the segmentation input. Finally, it was reported that lesion segmentation was poor when considering very small lesions.

Salem et al (45), proposed a FCNN in order to detect early T2-w lesions in MR images. Patient cohort included 60 early MS and CIS patients, with a baseline and a follow-up study for each patient, while new T2-w lesions were found in the follow-up exam. Authors concluded that the proposed methodology achieved increased accuracy regarding the detected newly formed T2-w lesions, and therefore it has the potential to be used in the clinical practice for monitoring disease progression.

Brown et al (46) implemented a FCNN for automatic segmentation of orbital fat, tested on pediatric-inset MS patients, while they introduced a preprocessing step of image calibration aiming to remove technical intensity artefacts. This method was evaluated on 1,018 scans form 256 participants. Study results report that automatic segmentations agreed with manual segmentation from an expert, reporting estimated mean Jacard index 0.74 , while the proposed image calibration contributed significantly in the performance of segmentation. Finally, the proposed methodology provided a robust serial calibration framework that allowed comparison of follow-up studies of the patient, while it is considered to be a fast technique that may be applied to large as well as to small datasets.

Ackaouy et al (47) proposed an unsupervised domain adaptation framework based on optimal transport (3D-Unet), Seg-JDOT, adapting a deep model to samples from a source domain to a target domain that share similar representations. This study used the MICCAI 2016 MS lesion segmentation challenge dataset (48) that contains 53 MRI images from MS patients, splitted to 15 train and 38 test images. Results from this study with single and multi-source training, indicated that model adaptation to a target site, can yield better model performance compared to the standard training.

Coronado et al (49) utilized a 3D CNN model based on multispectral MRI data of 1,006 RRMS patients, aiming to automatically segment gadolinium-enhanced T1 lesions. The dice similarity coefficient, true-positive rate, false-positive rate indexes over all the enhancing lesions were 0.77/0.90/0.23 when using FLAIR, T2 and pre- and post-contrast T1-w images, $0.72 / 0.86 / 0.31$ when using only pre and post contrast T1-w images and comparable performance when using only post-contrast images.
La Rosa et al (50) proposed a framework for automatic segmentation of cortical and white matter lesions based on T1 and T2-weighted MRI data, in a cohort of 90 MS patients including 728 gray and 3,856 white matter lesions. For this purpose, an FCNN architecture based on the 3D-U-Net was used. Results of the present study reported that the proposed framework was able to achieve a detection rate of $76 \%$ for both cortical and white matter lesions with a false positive rate of $29 \%$ in comparison to manual segmentation. Finally, it is reported that the proposed methodology achieved to adequately generalize the exams acquired in two hospitals with different scanners.

Gessert et al (51) examined a CNN model for lesion segmentation from two time points, using two path architectures. They stratified a cohort of 89 MS patients, with two MRI exams each, baseline and follow-up respectively, while for validation and testing they used a 3-fold cross-validation. Results indicate that the proposed model outperformed classic methodologies, while it was reported a lesion-wise false-positive rate of $26.4 \%$ at a true-positive rate of $74.2 \%$.

Essa et al (52) proposed an automatic segmentation framework, based on region-based CNN (R-CNN) model applied on T2-w and FLAIR MR images, evaluated on the MICCAI 2008 MS challenge (http://www.ia.unc.edu/MSseg). The proposed model shows competitive results compared with the state-of-the-art MS segmentation methods, with average total score 83.25 and average sensitivity $61.8 \%$ on the testing set.

Finally, Barquero et al (53) developed a CNN architecture (RimNet) for automated rim lesion detection in MS. MR imaging data were acquired from three different scanners, in a cohort of 124 patients. The multimodal RimNet architecture achieved to better classify lesions [area under curve $($ AUC $)=0.943]$ compared to unimodal approaches, while sensitivity and specificity (70.6 and $94.9 \%$, respectively) were comparable to the experts scores

\section{Classification: Diagnosis}

During the last years, DL techniques have been successfully applied for the diagnosis and classification of MS disease (54). These techniques can identify patterns of the imaging data without using an explicit feature extraction method, but instead using automatic feature extraction. Advantages stemming from the use of DL techniques in MS diagnosis include the increment of diagnostic accuracy, early stage diagnosis, increase the reliability of the diagnosis, decrease disease related cost, and improvement in patient quality of life. In this section, the most significant research articles in the MS classification using DL techniques are reviewed.

Yoo et al (55) presented an automatic framework for detection of MS pathology at an early stage. In this approach, 3D image patches extracted from myelin maps and the corresponding T1-w MR images was used to train a latent joint myelin feature representation using an unsupervised four-layer deep belief network (DBN) framework. This framework used 55 RRMS patients and 44 healthy controls, while by using 11-fold cross validation, it achieved $87.9 \%$ average classification accuracy, and authors reported that suggested method was able to identify MS image features from normal appearing brain tissues. 
Wang et al (56) implemented a 14-layer CNN combined with batch normalization, dropout and stochastic pooling techniques aiming towards early diagnosis and treatment of MS disease. Imaging data for the current study, included $38 \mathrm{MS}$ patients from eHealth laboratory and 26 healthy controls (HC). Results reported that the proposed 14-layer CNN network had sensitivity of $98.77 \%$, specificity $98.76 \%$ and accuracy 98.77\%, while after comparison of the aforementioned method with maximum pooling, average pooling, five traditional AI methods and a DL method, it was shown that the proposed method outperforms all the above-mentioned techniques.

Zhang et al (57) developed a DL algorithm based on CNN that combined parametric rectified linear unit (PReLU) and dropout techniques, in order to accurately separate MS from HC. This study stratified 676 MS brain slices and $681 \mathrm{HC}$ slices, while data augmentation technique was used in order to increase the size of the training set. The 10-layer deep convolutional neural network used in this study was consisted of 7 convolution layers and 3 fully-connected layers. The proposed method achieved $98.22 \%$ sensitivity, $98.24 \%$ specificity and $98.23 \%$ accuracy, while the dropout method increased accuracy by $0.88 \%$, PReLU increased accuracy by $1.92 \%$ compared to ordinary ReLU and by $1.48 \%$ compared to leaky ReLU.

Talo et al (58) implemented a deep transfer learning framework to automatically classify normal and abnormal brain MR images via using a ResNet34 CNN DL model featuring data augmentation, optimal learning rate finder and fine-tuning to adapt a pre-trained model. This study stratified a cohort of 42 subjects (2 HC and 40 patients with cerebrovascular, neoplastic, degenerative, and inflammatory disease types), resulting in 613 images, 27 normal and 513 abnormal, for training and validation. Results from the current study report that they achieved $100 \%$ classification accuracy.

Lu et al (59) presented a transfer learning technique for automatic detection of pathological brain, by utilizing a pre-trained model featuring AlexNet architecture. In this study brain MR images from $38 \mathrm{HC}$ and 177 pathological were used, including pathologies such as Alzheimer, Glioma, Huntington, AIDS dementia, MS, and Pick. Results reported that the proposed method achieved accuracy of $100 \%$ which, outperformed state-of-the-art approaches.

McKinley et al (60), investigated the ability of DL algorithm to discriminate radiologically progressive from radiologically stable patients, in 3 different private datasets. To this end, DeepSCAN MS classifier, a fully-convolutional neural network was utilized. The methodology that was used in this study, achieved to temporally track lesion load changes by leveraging measures of uncertainty in the location of lesion boundaries. Results from the present study, disclosed that the proposed framework was able to separate progressive from stable time-points (AUC $=0.999)$ and changes in lesion volume $(\mathrm{AUC}=0.71)$. Moreover, method validation on two external datasets confirmed the performance of this method by achieving accuracies 75 and $85 \%$ in separating stable and progressive time-points.

Marzullo et al (61) introduced a graph convolutional neural network (GCNN) toclassify MS patients on fourclinical profiles, (CIS, RR, secondary-progressive SP, primary-progressive PP). Methodology in the present study used structural connectivity information by DWI and evaluated the classification performance using unweighted and weighted connectivity matrices. Moreover, the role of graph-based features aiming to better characterization and classification of the MS pathology were investigated. For the purposes of this study, $90 \mathrm{MS}$ patients (12 CIS, 30 RRMS, 28 SPMS and 20 PPMS) and 24 healthy controls were included in the analysis. Results demonstrated that the aforementioned NN framework offers very good performance in clinical profiles classification, while graph weights representation of brain connections offer significant information for clinical profiles discrimination.

Eitel et al (62) presented a DL framework based on a 3D $\mathrm{CNN}$ and a layer-wise relevance propagation (LRP) in order to accurately diagnose MS disease. In this study, LRP was used as an additional tool aiming to reveal further relevant image features that will be afterwards forwarded to the trained $\mathrm{CNN}$. The CNN model was trained using MRI data from the Alzheimer Disease Neuroimaging Initiative (921 patients) and afterwards the CNN was used to discriminate among MS (76 patients) and controls (71 healthy controls). Authors reported that the presented methodology showed accuracy $87 \%$ and AUC 96\%, concluding that LRP in conjunction with proposed CNN is a framework capable of sufficiently classify MS patients and healthy controls.

Narayana et al (63) used a CNN in order to evaluate the performance in predicting enhanced MR lesions without using contrast agents. To this end, a CNN was used for classifying lesions from MR images as enhanced or unenhanced. In this study, 1,008 MS patients were involved, while at least one enhancing lesion was observed for 519 participants. MR demyelinating lesions were classified in two classes, enhanced and unenhanced, while performance was assessed by using fivefold cross-validation. Results report sensitivity $78 \%$, specificity $73 \%$, AUC 0.82 for slice-wise prediction, while for participant-wise these were $72 \%, 70 \%$ and 0.75 , respectively.

Maggi et al (64) proposed a DL based prototype for automated assessment of the central vein sign (CVS) in WM MS lesions. This study included 80 subjects from three different sites, 42 MS, 33 MS mimics with diseases that are described by WM abnormalities similar to MS, and 5 patients with uncertain diagnosis. To this end, a 3D CNN ('CVSnet') was designed, trained on 47 examinations and tested on the remaining 33. Authors reported that the proposed framework achieved human expert performance, achieving lesion-wise median balanced accuracy of $81 \%$ and subject-wise balanced accuracy of $89 \%$ on the validation and $91 \%$ on the test set, while evaluation on data from different hospitals-scanners is promising for larger multi-center trials using the CVS marker in the MS diagnostic criteria.

Wang et al (65), utilized a CNN framework in order to discriminate neuromyelitis optical spectrum disorder (NMOSD) (41 patients) from MS (47 patients), a challenging classification problem considering that NMOSD is a rare disease thus there is a limitation in the availability of exams, as well as the lesions in the above mentioned diseases are scattered and overlapping, adding an extra embodiment in the classification procedure. Study results reported that the novel proposed CNN model achieved to better discriminate NMOSD from MS compared to conventional CNN models. More specifically, the proposed method exhibited accuracy 0.75 , 
sensitivity 0.707 , and specificity 0.759 , while traditional $3 \mathrm{D}$ CNNs that tested on the same problem did not achieve to distinguish the different classes.

Roca et al (66) used a DL network in order to predict the expanded disability status scale (EDSS) in a cohort of 1,446 MS patients, partitioned in 971 subjects for training, and 475 subjects for testing. The proposed framework consisted of a CNN model and a classical ML predictor. They finally reported that the proposed method was able to predict two-year EDSS score, based on FLAIR MRI imaging data, while the more informative variables were age, volume of lateral ventricles and the lesion load in main white matter tracts.

Lopatina et al (67) utilized a CNN model aiming to identify MS patients by analyzing susceptibility weighted images, considering that in this protocol vein patterns are identifiable and it is also able to indicate extensive demyelination and iron accumulation. The stratified cohort was composed by 66 MS patients and 66 healthy controls, while results indicate that veins located in the anterior medial and lower peripheral regions, are most relevant for the classification decision.

\section{Post processing techniques and image enhancement methods}

Reducing rescans and recalls is of great importance for the optimization of health care management, since low image quality MR acquisitions often need to be repeated, thus leading to increased hospital costs and extended MR examination times. Furthermore, visual inspection of the related examinations aiming to assess image quality, is impractical in large multi-center studies as well as prone to errors emanating from intra-observer variability. In recent years, computational tools that assess the image quality of the examinations have been developed based on automated assessment of image quality metrics. To this purpose, computer algorithms have been implemented during the last years, aiming to automatically assess image quality as well as acquisition parameters. In this section, recent studies focusing on solving the aforementioned problems by using DL techniques in MR imaging of neurodegenerative diseases are reviewed.

Sreekumari et al (68) developed an automated method for assessing the need of rescan, in motion corrupted brain scans. Authors developed a CNN with 7 convolutional layers, 4 max pooling layers, and 3 batch normalization layers that computed the probability for a MR series to be clinically useful, while by combining this probability with a scan dependent and radiologist defined threshold, they determined whether a series need to be rescanned. Moreover, the classification performance was compared with that of 4 technologists and 5 radiologists in 49 series, stemming from MS and stroke patients, characterized by low and moderate motion artefacts. Results indicated that radiologists - technologists produced mean ratio of rescans/recalls of (4.7_5.1)/(9.5_6.8) for MS and (8.6_7.7)/(1.6_1.9) for stroke, while DL produced (7.3_2.2)/(3.2_2.5) for MS, and (3.6_1.5)/(2.8_1.6) for stroke, concluding that this technology independent method can reliably decrease rescan and recall rates.

Sujit et al (69) developed a DCNN aiming to automatically evaluate the quality of multicenter structural brain MRI images, using 1,064 images from autism patients from ABIDE database (60\% training, $20 \%$ validation and $20 \%$ test) while they tested on a cohort of 110 MS patients from the CombiRx dataset (70). The results demonstrated the high accuracy of the proposed method to evaluate image quality of structural brain MRI in multi-center studies (ABIDE dataset achieved AUC 0.90 , sensitivity 0.77 , specificity 0.85 , accuracy 0.84 , PPV 0.42, and NPV 0.96 while for the CombiRx there were AUC 0.71 , sensitivity 0.41 , specificity 0.84 , accuracy 0.73 , PPV 0.48, and NPV 0.80).

In many applications, the compromise between spatial resolution, SNR, as well as temporal resolution in specific protocols, is limiting the clinical and research applicability of the MR modality. An ordinary approach to overcome this problem is to acquire images with adequate in-plane resolution and low through-plane resolution and afterwards interpolate data, by using super-resolution techniques, in order to obtain isotropic voxels. To this purpose, Zhao et al (71) developed a DL method, called SMORE, that accomplished both anti-aliasing and super-resolution using no external atlases. Authors demonstrated the performance of the proposed algorithm in four applications: i) improve visualization of the brain WM lesions in MS patients; ii) improve the visualization of scarring in cardiac left ventricular remodeling after myocardial infarction; iii) performance on multi-view images of the tongue; and iv) improve performance in brain ventricular system parcellation. Regarding the MRI visualization of MS lesions, that is speculated in the present review article, authors examined whether the SMORE computationally enhanced FLAIR images can provide additional diagnostic information compared to a conventional interpolation. For this purpose, FLAIR data was reconstructed from initial resolution of $0.828 \times 0.828 \mathrm{~mm} \times 4.4 \mathrm{~mm}$ onto a $0.828 \times 0.828 \mathrm{~mm} \times 0.828 \mathrm{~mm}$ digital grid by a conventional b-spline interpolation, the JogSSR approach that is a super resolution method which improves resolution in the through-plane direction (72), as well as the proposed SMORE algorithm. Authors reported that both JogSSR and SMORE resulted in sharper edges compared to the conventional b-splines interpolation, while SMORE results appeared to be more realistic, however results in this part of the study were assessed only by visual inspection while authors do not provide any quantitative measurement.

Another aspect that has recently gained interest, is the generation of synthetic MR images by using computational techniques. This is an interesting and emerging area of research especially considering that some protocols may be time-consuming and some sequences may be missed due to limited scanning time or patients' interruptions in case of anxiety and confusion. Also, the ability to synthesize information related to different imaging modalities directly from MRI has gained the attention of many researchers in the field of DL in medical imaging.

To this purpose, Wei et al (73) used a 3D FCNN to predict FLAIR pulse sequence from other MRI protocols. For this study, 20 MS patients and 4 healthy controls were involved, including T1-w, T2-w, PD, FLAIR, T1 SE and double inversion echo sequences. The performance of the proposed method was compared qualitatively and quantitatively with four state-of-the-art approaches: modality propagation (74), random forests including 60 trees (75), U-Net (76) and voxel 
wise multilayer perceptron consisted of two hidden layers and 100 hidden neurons for every layer. Results from this study indicated that the proposed FLAIR synthesis method provides competitive performance to previous techniques. In more detail, the proposed technique was statistically significantly better than the other methods $(\mathrm{P}<0.05)$ in average, by providing MSE (SD) 918.07 (41.70) and SSIM(SD) 0.860 (0.031). Finally, authors compared the aforementioned methods in a MS lesion detection task, by evaluating the MS lesion to NAWM as well as to the surrounding NAWM tissue contrast. Also, in this task the proposed method outperformed the competitive techniques on both ratios, revealing that it provides better contrast for MS lesions.

Salem et al (77) proposed a synthesis method for MS lesions on MR images aiming to improve the performance of supervised machine learning algorithms, thus avoiding the lack of ground truth. To this end, they used a two-input two-output FCNN for MS lesion synthesis, in which the lesion information was encoded as discrete binary intensities level masks. For this purpose, authors assessed the proposed methodology in two cohorts of patients, by evaluating the similarities among real and synthetic images, as well as by measuring the performance of lesion detection by segmenting both the original and the synthetic images. The two cohorts used included $15 \mathrm{HC}$ and 65 patients with CIS or RRMS, and the ISBI2015 dataset (24) comprising 5 training and 14 testing subjects with 4 to 5 follow-up studies for every subject, while they also used data augmentation techniques. The effect of data augmentation was demonstrated in both analyzed datasets, while results showed the effectiveness of using synthetic MS lesions. Finally, regarding the ISBI2015 challenge, the model was trained using a single image and the synthetic data augmentation and performed similarly to other CNN methods that were fully trained.

Wei et al (78) proposed a method to predict positron emission tomography (PET)-derived myelin content map from multimodal MR imaging data, by introducing a new approach called Sketcher-refiner GANs with a specially designed adversarial loss function, in which the Sketcher network generated global anatomical and physiological information, while the Refiner model refined and generated the tissue myelin content. For this purpose, a dataset including $18 \mathrm{MS}$ patients and $10 \mathrm{HC}$ was used, while both MR and PET images were available. It was reported that regarding image quality and myelin content, the proposed approach outperformed the state-of-the-art methods, as well as that prediction results were comparable to PET derived gold standard both in global and voxel-wise levels.

Finck et al (79) implemented a diamond shape topology generative adversarial NN (DiamondGAN) in order to produce synthetic double inversion recovery (synthDIR) images while the diagnostic performance of the proposed images was compared to conventional MR images. This study recruited 100 MS patients, while the DiamondGAN was trained in a subset of 50 MS patients and an additional 50 images of generated synthetic data. Results indicate that synthetically generated DIR images resulted in improved detection of juxtacortical lesions, leading to improvement on lesion detection.

Finally, the last category is related to computational methods that are focusing on post-processing MR images for image reconstruction, and computational analysis. These methods are of great interest considering that they can bypass unreliable computational methods and ill-conditioned mathematical problems, as well as combine large-scale information from multi-site studies by providing quantitatively and qualitatively consistent images to automated algorithms.

Yoon et al (80) presented a DL network (QSMnet) with a modified U-net structure able to generate high quality susceptibility source map from single orientation data. A total of 12 healthy controls were used for training, using COSMOS QSM maps, while for evaluation data from a microbleed, a MS and a patient with intraparenchymal hemorrhage were used. Results demonstrated that QSMnet provided superior image quality results compared to TKD and MEDI, while the image quality is comparable to COSMOS.

Bollman et al (81) trained a FCNN, DeepQSM, in order to invert the magnetic dipole kernel convolution, and to provide a QSM framework that determines the composition of myelin sheaths of nerve fibers in the brain, as well as to assess quantitative information on iron homeostasis and its dysregulation. This network was trained on synthetic examples, while for testing procedure there were performed four experiments with synthetic data, a single orientation background field corrected tissue phase image and an STI susceptibility map, and finally clinical data from a patient with MS. Study results showed that the proposed methodology enabled identification of deep brain structures, not visible in MRI data. Furthermore, study results revealed that the presented methodology provides information for magnetic tissue properties, and finally showed increased sensitivity in identifying WM lesions regarding the MS patient.

Dewey et al (82) proposed a contrast harmonization method, DeepHarmony, by using U-Net DL technique in order to provide images with consistent contrast. To this end, 12 subjects (10 MS and $2 \mathrm{HC}$ ) were scanned twice within 30 days, and longitudinal data were retrospectively collected from 45 RRMS patients, while both cohorts were based on two different scanning protocols. Results suggested that DeepHarmony harmonized images showed significant improvement, as well as reduced dependency of atrophy calculations when using DeepHarmony method.

Liu et al (83) implemented a fast myelin water fraction (MWF) maps data analysis method, that was based on Myelin Water Imaging data acquired by a 32-echo 3D gradient and spin echo sequence. For this purpose, an NN model was used on data stemming from 4 healthy controls, while for testing there were utilized $1 \mathrm{MS}$ brain, 1 healthy spinal cord, and 2 healthy brains acquired from a different scanner. Results proposed that time for calculation of MWF maps was dramatically reduced while their quality was similar to ground truth levels.

\section{Discussion}

This review paper aims to shed light on DL applications related to MR imaging in MS and CIS. For this purpose, an extensive search led to 45 original research papers focused on this subject, that were further grouped in three categories i.e. segmentation, classification and a broader category related to image optimization and post processing techniques in MS and CIS. Findings of the current review indicate that there is 
Table I. Summary and performance metrics of the reviewed publications relevant to detection-segmentation tasks.

\begin{tabular}{|c|c|c|}
\hline Author (Ref.) & Year & Performance \\
\hline Valverde et al (22) & 2017 & $\begin{array}{l}\text { Competition score } 87.12 \\
\text { Volume difference down to } 40.8 \\
\text { TPR up to } 68.7\end{array}$ \\
\hline Birendaum et al (23) & 2017 & $\begin{array}{l}\text { Dice similarity } \\
\text { coefficient } 0.627\end{array}$ \\
\hline Gros et al (25) & 2019 & DSC up-to 0.604 \\
\hline Aslani et al (28) & 2019 & $\begin{array}{l}\text { DSC up-to } 0.611 \text { (ISBI) } \\
\text { DSC up-to } 0.6655 \text { (private) }\end{array}$ \\
\hline Sander et al (29) & 2019 & $\begin{array}{l}\text { Mean } \% \text { change/SD test- } \\
\text { retest } 0.45 \% / 0.005 \text { (brainstem) } \\
\text { Dice score/SD } 0.97 / 0.005 \text { for } \\
\text { brainstem, } 0.95 / 0.0131 \text { for } \\
\text { mesencephalon, } 0.98 / 0.006 \\
\text { for pons, } 0.95 / 0.015 \text { for medulla } \\
\text { oblongata }\end{array}$ \\
\hline Hashemi et al (31) & 2019 & DSC 0.703 \\
\hline
\end{tabular}

MD-GRU

CNNs based on the single view CNN (V-Net) and the longitudinal network (L-Net)

A cascade of two 3D

patch-wise CNNs

A sequence of two CNNs

Deep end-to-end 2D CNN

3D FCNN (FC-DenseNet)

\section{FCNN}

DL CNN method nicMSlesions

A cascade of two convolutional neural networks CNNs

\section{FCNN}

3D CNN WITH dropout

Nair et al (42) $\quad 2020$ TPR 0.8, FDR 0.2

McKinley et al (43) 2020 DSC 0.661

Narayana et al (44)
Two state-of-the-art CNNs architectures, a 3D Unet consisting of a reference implementation and a more recently proposed architecture, the DeepSCAN U-net, a fully CNN
2020

DSC up-to 0.91 (FLAIR)

DSC up-to 0.90

(FLAIR, PD, T1, T2)

DSC up-to 0.60 (PD)
Dataset, \#patients (training/test)

45 patients with MS

from MICCAI 2008 dataset, and two private MS clinical datasets 2015 Longitudinal Multiple Sclerosis Segmentation Challenge

1,042 subjects; 459 HC, $471 \mathrm{MS}$, 112 with other spinal pathologies

$37 \mathrm{MS}$ private dataset, and 14 patients with MS from ISBI 2015 longitudinal MS lesion segmentation challenge dataset 50 patients with MS and $17 \mathrm{HC}$, 20 independent patients with MS, 50 patients with Alzheimer's

15 patients from the MSSEG of the 2016 Medical Image Computing and Computer Assisted Intervention conference, five patient data from the longitudinal MS lesion segmentation challenge of ISBI conference 1,000 patients with RRMS from the CombiRx clinical trial 14 patients with MS

122 MRI exams from patients with RRMS (90 patients for training and validation of the classifier, 32 patients for testing), while a second dataset including 10 MRI datasets was used for direct comparison with other centers

1,008 patients with clinically definite MS

1,064 patients with RRMS

2016 MSSEG training dataset, retrained on a larger dataset comprising of 122 patients with MS (50 training, 40 validation and 32 patients for testing)

1,000 patients with MS 
Table I. Continued.

\begin{tabular}{|c|c|c|c|c|}
\hline Author (Ref.) & Year & Performance & Architecture & Dataset, \#patients (training/test) \\
\hline Salem et al (45) & 2020 & $\begin{array}{l}\text { DSC for detection } 0.83, \\
\text { DSC for segmentation } 0.55\end{array}$ & FCNN & $\begin{array}{l}60 \text { early patients with } \mathrm{MS} \text { and } \\
\text { CIS }\end{array}$ \\
\hline Brown et al (46) & 2020 & Not applicable. & FCNN similar to U-net & $\begin{array}{l}1,018 \text { scans form } 256 \text { participants } \\
\text { in a study of pediatric-onset MS }\end{array}$ \\
\hline Ackaouy et al (47) & 2020 & $\begin{array}{l}\text { No table with absolute } \\
\text { measurements, too many } \\
\text { combinations of multi-centric } \\
\text { experiments, only boxplot results }\end{array}$ & 3D-Unet & $\begin{array}{l}53 \text { patients with MS from } \\
\text { MICCAI } 2016 \text { MS lesion } \\
\text { segmentation challenge }\end{array}$ \\
\hline Coronado et al (49) & 2020 & DSC 0.77 & $3 \mathrm{D} \mathrm{CNN}$ & 1,006 patients with RRMS \\
\hline La Rosa et al (50) & 2020 & DSC 0.62 & $\begin{array}{l}\text { FCNN architecture based } \\
\text { on the 3D U-Net }\end{array}$ & 90 patients with MS \\
\hline Gessert et al (51) & 2020 & DSC up-to 0.656 & $\mathrm{CNN}$ & 89 patients with MS \\
\hline Essa et al (52) & 2020 & $\begin{array}{l}\text { VD down to } 0.451, \text { TPR } 0.681 \text {, } \\
\text { FPR } 0.632\end{array}$ & Region-based CNN & $\begin{array}{l}45 \text { patients with MS from } \\
\text { MICCAI } 2008\end{array}$ \\
\hline Barquero et al (53) & 2020 & DSC 0.835 & CNN (RimNet) & 124 patients with MS \\
\hline
\end{tabular}

CNNs, convolution neural networks; MS, multiple sclerosis; MD-GRU, multi-dimensional gated recurrent units; SD, standard deviation; HC, healthy controls; DSC, Dice Similarity Coefficient; ISBI, International Symposium on Biomedical Imaging; TPR, true positive rate; FCNN, fully convolutional neural network; MSSEG, MS lesion segmentation challenge; RRMS, relapsing-remitting multiple sclerosis; SN, sensitivity; MRI, magnetic resonance imaging; FLAIR, fluid-attenuated inversion recovery; PD, proton density; CIS, clinically isolated syndrome; MICCAI, medical image computing and computer assisted intervention society; VD, volume difference.

a growing interest during the last years towards applying DL techniques for segmentation and classification related to MS imaging studies.

A performance analysis was conducted by grouping the studies into three main categories based on imaging tasks such as segmentation in Table I, classification in Table II and various image processing tasks (synthesis, quality assessment, image enhancement, etc) in Table III. A few important issues in comparing these studies namely are: i) the lack of a clear data stratification protocol (57-59); ii) applying 'good practices' such as use of separate internal and external validation sets (28); iii) incorporating an adequate number of patients for DL model convergence $(35,83)$; iv) evaluating with appropriate metrics $(42,59)$ like AUC in addition to ACC in binary classification tasks; and v) incorporating different metrics for the same task such as DSC (23) versus VD (22) in segmentation. Thus, reporting the aforementioned performance measurements was a difficult task which included interpreting figures, highly variable experimental settings and, most importantly, identifying metrics on unseen testing sets (if available) versus internal validation sets which were also used in optimizing the models. Nevertheless, Narayana et al (44) proposed a FLAIR-based lesion segmentation with a testing DSC of 0.91 and Sander et al (29) utilized a multi-dimensional gated recurrent unit model achieving a performance up-to DSC 0.97. In MS classification, McKinley et al (60) achieved sensitivity up-to 0.72 on two different external validation sets, similar to Narayana et al (63) (SN 0.72) on patient-basis versus SN 0.78 on a slice-basis in the same study.

Concerning the limitations of the reviewed papers, the most pronounced are associated with the absence of ground truth, the small available cohorts, and the lack of generalization of the results in multi-center data from diverse vendors, in Fig. 4 presents the limitations of the reviewed literature grouped in a chart pie. Finally, another noticeable limitation of DL techniques is the lack of explainability, which is particularly critical for the clinical translation of such methods to clinical practice as well as for adding precision in MS and CIS diagnosis and disease management.

Considering the segmentation procedure, the fundamental MRI protocols, T1-w, T2-w and PD can be characterized based on their advantages concluding that, T1-w images enclose detailed anatomical information and thus facilitate image registration tasks involved in e.g. development of brain atlases. On the contrary, T2-w images are beneficial for highlighting WM lesions which appear as bright areas, thus assisting lesion detection tasks. However, the main drawback of these techniques is the insufficient differentiation of CSF, GM and lesions in terms of image contrast. Finally, PD-w images are beneficial in revealing MS lesions as areas of increased contrast compared to WM, even better than T2-w, but still the relative image contrast between MS lesions and CSF are in the same range of intensities. A major limitation for accurate image segmentation is pixel misclassification due to the partial volume effect generated from the low spatial resolution of the MR protocols which renders difficult the accurate delineation of brain regions and decreases the accuracy of volumetric studies. Finally, inter-observer variability is a major problem when trying to generate ground truth label data, while some of the factors that may hamper this task include image quality, different levels of user expertise and domain knowledge. Concerning the reviewed research studies, in Narayana et al (41) segmentation results were assessed by 
Table II. Summary and performance metrics of the reviewed publications relevant to classification tasks.

\begin{tabular}{|c|c|c|c|c|}
\hline Author (Ref.) & Year & Performance & Architecture & Dataset, \#patients (training/test) \\
\hline Yoo et al (55) & 2018 & $\begin{array}{l}\text { ACC } 0.879, \text { SN } 0.873 \\
\text { SP } 0.886, \text { AUC } 0.88\end{array}$ & $\begin{array}{l}\text { Unsupervised } \\
\text { four-layer DBN }\end{array}$ & $\begin{array}{l}55 \text { patients with RRMS and } \\
44 \mathrm{HC}\end{array}$ \\
\hline Wang et al (56) & 2018 & $\begin{array}{l}\text { ACC 0.988, SN 0.988, } \\
\text { SP } 0.988\end{array}$ & $\begin{array}{l}\text { 14-layer CNN combined } \\
\text { with batch normalization, } \\
\text { dropout and stochastic } \\
\text { pooling techniques }\end{array}$ & $\begin{array}{l}38 \text { patients with MS from } \\
\text { eHealth laboratory and } 26 \mathrm{HC}\end{array}$ \\
\hline Zhang et al (57) & 2018 & $\begin{array}{l}\text { ACC 0.982, SN 0.982, } \\
\text { SP } 0.982\end{array}$ & $\begin{array}{l}\text { 10-layer deep convolutional } \\
\text { neural network used in } \\
\text { this study was consisted of } \\
7 \text { convolution layers and } \\
3 \text { fully-connected layers }\end{array}$ & $\begin{array}{l}676 \mathrm{MS} \text { brain slices and } \\
681 \mathrm{HC} \text { slices }\end{array}$ \\
\hline Talo et al (58) & 2019 & $\begin{array}{l}\text { Stage-1: ACC } 0.979, \text { SN } 0.778 \text {, } \\
\text { Stage-2: ACC } 0.979, \text { SN } 0.74 \\
\text { Stage-3: ACC } 1, \text { SN } 1\end{array}$ & CNN based ResNet34 & $\begin{array}{l}42 \text { subjects ( } 2 \mathrm{HC} \text { and } 40 \\
\text { patients with cerebrovascular, } \\
\text { neoplastic, degenerative and } \\
\text { inflammatory disease types) }\end{array}$ \\
\hline Lu et al (59) & 2019 & ACC 1 & $\begin{array}{l}\text { AlexNet structure and } \\
\text { stochastic gradient descent } \\
\text { with momentum transfer } \\
\text { learning technique }\end{array}$ & $\begin{array}{l}\text { MR images from } 38 \mathrm{HC} \text { and } \\
177 \text { pathological, including } \\
\text { pathologies such as Alzheimer's, } \\
\text { glioma, Huntington's, AIDS } \\
\text { dementia, MS and Pick's }\end{array}$ \\
\hline McKinley et al (60) & 2019 & $\begin{array}{l}\text { External validation sets: } \\
\text { Zurich: ACC 0.75, SN } 0.60 \\
\text { Munich: ACC 0.85, SN } 0.72\end{array}$ & $\begin{array}{l}\text { DeepSCAN MS classifier, } \\
\text { a fully-convolutional } \\
\text { neural network }\end{array}$ & $\begin{array}{l}\text { Bern (train-test), Zurich and } \\
\text { Munich datasets }\end{array}$ \\
\hline Marzullo et al (61) & 2019 & F1 0.74, PR 0.76, RC 0.75 & Graph CNN & $\begin{array}{l}90 \text { patients with MS (12 CIS, } \\
30 \text { RRMS, } 28 \text { SPMS and } \\
20 \text { PPMS) and } 24 \mathrm{HC}\end{array}$ \\
\hline Eitel et al (62) & 2019 & ACC 0.87 , AUC 0.96 & 3D CNN and a LRP & $\begin{array}{l}921 \text { patients with Alzheimer's } \\
\text { disease for training, validation in } \\
76 \mathrm{MS} \text { and } 71 \mathrm{HC}\end{array}$ \\
\hline Narayana et al (63) & 2020 & $\begin{array}{l}\text { Slice: AUC 0.82, SN 0.78, } \\
\text { SP } 0.73 \\
\text { Patient: AUC 0.75, SN 0.72, } \\
\text { SP } 0.70\end{array}$ & CNN (fully connected) & 1,008 participants with MS \\
\hline Maggi et al (64) & 2020 & $\begin{array}{l}\text { ACC 0.91, SN 0.89, SP } 0.92 \\
\text { Vesselness: ACC 0.69, } \\
\text { SN 0.61, SP } 0.77\end{array}$ & 3D CNN ('CVSnet') & $\begin{array}{l}42 \mathrm{MS}, 33 \mathrm{MS} \text { mimics and } \\
5 \text { patients with uncertain diagnosis }\end{array}$ \\
\hline Wang et al (65) & 2020 & ACC 0.75, SN 0.707, SP 0.759 & $\mathrm{CNN}$ & $\begin{array}{l}41 \text { patients with NMOSD and } 47 \\
\text { patients with MS }\end{array}$ \\
\hline Roca et al (66) & 2020 & MSE 3 & $\mathrm{CNN}$ & $\begin{array}{l}\text { 1,446 MS ( } 971 \text { training, } \\
475 \text { testing) }\end{array}$ \\
\hline Lopatina et al (67) & 2020 & ACC up-to 0.95 (echo 1) & $\mathrm{CNN}$ & $66 \mathrm{MS}$ and 66 healthy controls \\
\hline
\end{tabular}

SP, secondary progressive; AUC, area under curve; DBN, deep belief network; HC, healthy controls; CNN, convolution neural networks; MS, multiple sclerosis; MR, magnetic resonance; CIS, clinically isolated syndrome; LRP, layer-wise relevance propagation; NMOSD, neuromyelitis optical spectrum disorder; MSE, mean square error; ACC, accuracy; SN, sensitivity; RRMS, relapsing-remitting multiple sclerosis; PR, precision; RC, recall; SPMS, secondary progressive multiple sclerosis; PPMS, primary progressive multiple sclerosis.

two experts, while in the study from Brown et al (46) results were evaluated with manual segmentations stemming from only one expert.

Considering the classification related task, initially it can be reported that fundamentally this is not a binary problem. CIS diagnosis adds another level of complexity to the clas- sification problem, which can be treated as multiple binary comparisons, i.e., controls vs. MS, controls vs CIS, CIS vs MS, while for each problem a different classifier can be developed. Furthermore, considering the constantly increasing imaging databases, especially those including longitudinal data, research studies may shift to the development of CAD based 


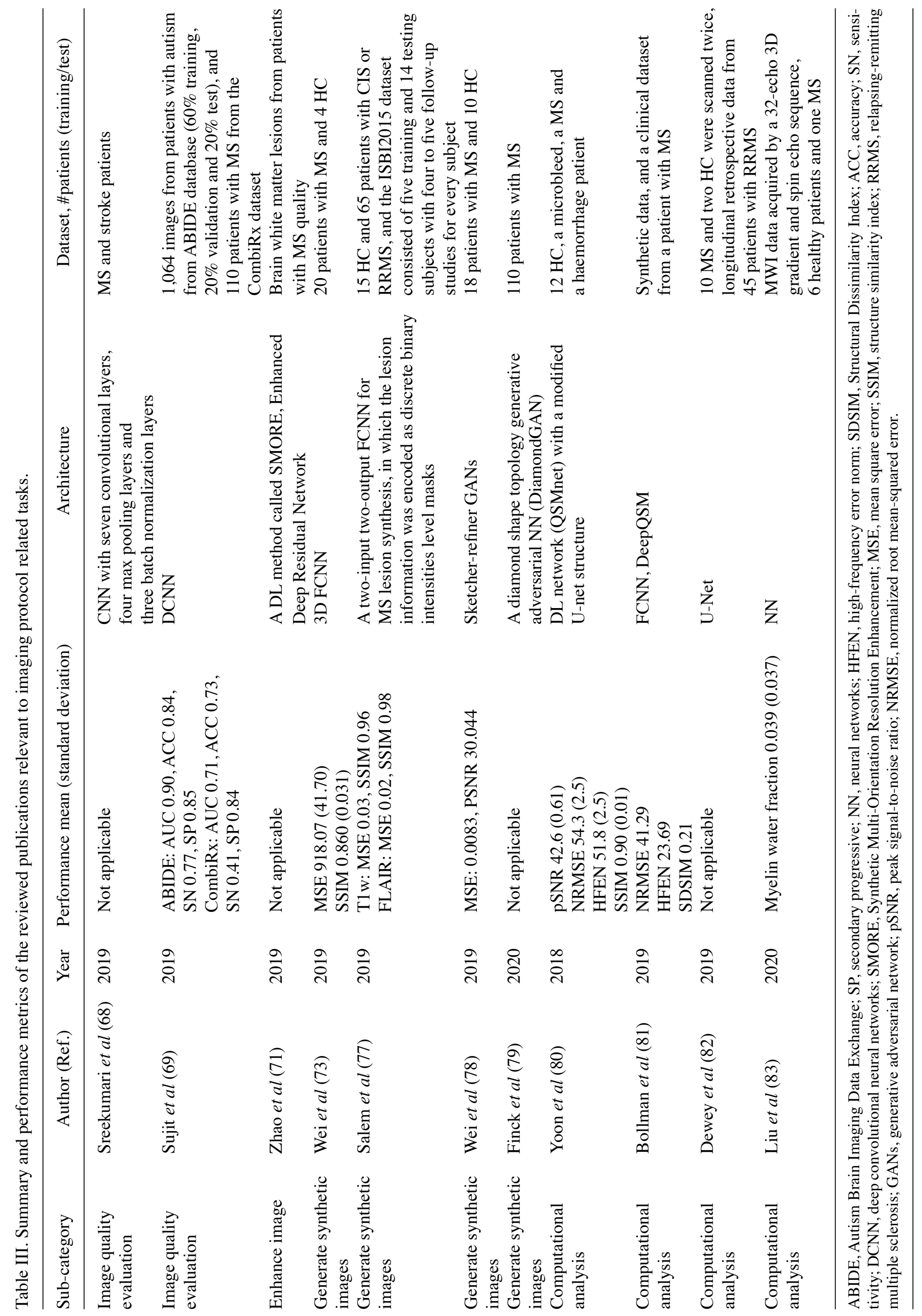




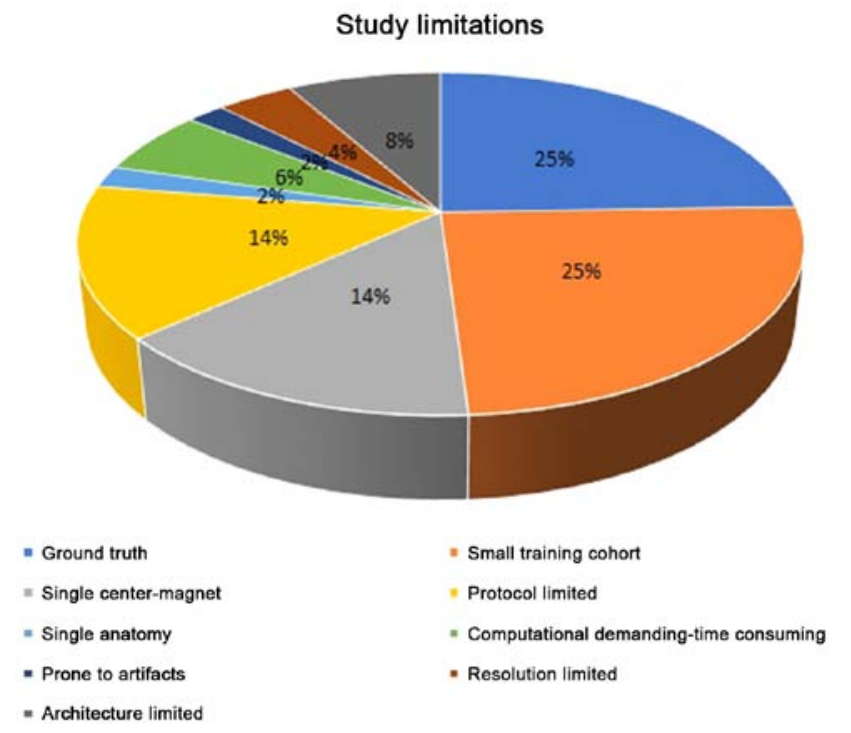

Figure 4. Study limitations of the relevant publications presented in a pie chart. Most common limitations in the reviewed research article were the small training cohort and the lack of ground truth.

systems for early detection of the MS disease. In addition, classification techniques can be grouped in class-based methods in which MS lesions are separated in WM, GM and CSF lesions, and outlier-based methods in which GM, WM, and CSF are distinct classes from MS lesions.

Furthermore, images stemming from different centers, scanners and protocols, provide a basis in order to build robust algorithms that can generalize the aforementioned tasks. However, in the small dataset setting, transfer learning techniques are methods used for alleviating the limited availability of training samples by tuning parameters of pre-trained networks with less data in order to adapt existing models in new domains and achieve higher level of generalization. In domain adaptation techniques, optimal transfer is established through a source and a target domain in order to deploy a model on the target site that was not included in the training process, and this is a promising research field for providing integrated solutions.

Moreover, according to best practices that must be followed in order to build a robust DL methodology, some research studies mentioned in this review article might not satisfy these prerequisites. More specific, Ackaouy et al (47) and Talo et al (58) methodology did not follow the universally accepted best practices in DL training, considering the data splitting. Data should be split into training set, validation set and testing set in order to ensure that the model will not be over-fitted. External validation set is necessary in order to enhance reproducibility and generalizability of a prediction model to new patients. In the reviewed research articles, only a few used external validation set in order to check reproducibility and enhance generalizability $(29,34,40,60,71)$.

Finally, data imbalance problem is very common in MS machine learning applications and can lead to a training network with sufficient high prediction but low recall, thus biased to the class with the most data. Techniques such as two-step training, sample re-weighting, balance sampling and similarity loss functions are promising for bypassing such problems, while they can, also, provide computational techniques suited to highly unbalanced problems.

Future DL research work on MS considering also the aforementioned limitations, should focus on three different directions. The first direction regards the formation of large and more diverse datasets, with optimized protocols, for providing a ground truth validation framework of existing and future techniques. The second direction concerns the need for MS imaging data harmonization techniques for reducing the inherent information heterogeneity of multi-centric data due to different vendors and protocols and enabling the development of more robust and accurate AI models. Finally, the development of CAD systems for early detection of MS disease is of great importance based on the increased availability of larger datasets. Such systems offering cloud-based AI services, can provide an enhanced diagnostic experience to clinicians and offer better diagnostic opportunities especially in rural areas that usually lack access to specialists. All these directions regarding the future of DL in MS have to promote explainability and trustworthiness, and not only target increased performance, in order to realize the vision of real-world use of AI algorithms in clinical practice.

\section{Acknowledgements}

Not applicable.

Funding

No funding was received.

\section{Availability of data and materials}

Not applicable.

\section{Authors' contributions}

EEK, EP and KM conceived the designed study. EEK, EP, ET and KM researched the literature, performed analysis and interpretation of data and drafted the manuscript. EEK, EP, ET, TGM, PS, GZP, AT, DAS, AK and KM critically revised the article for important intellectual content. All authors have read and approved the final manuscript. Data sharing is not applicable.

\section{Ethics approval and consent to participate}

Not applicable.

\section{Patient consent for publication}

Not applicable.

\section{Competing interests}

DAS is the Editor-in-Chief of the journal, but had no personal involvement in the reviewing process, or any influence in terms of adjudicating on the final decision for this article. All the authors declare that they have no competing interests. 


\section{References}

1. Ortiz GG, Pacheco-Moisés FP, Macías-Islas MÁ, Flores-Alvarado LJ, Mireles-Ramírez MA, González-Renovato ED, Hernández-Navarro VE, Sánchez-López AL and Alatorre-Jiménez MA: Role of the blood-brain barrier in multiple sclerosis. Arch Med Res 45: 687-697, 2014.

2. Lopes Pinheiro MA, Kooij G, Mizee MR, Kamermans A, Enzmann G, Lyck R, Schwaninger M, Engelhardt B and de Vries HE: Immune cell trafficking across the barriers of the central nervous system in multiple sclerosis and stroke. Biochim Biophys Acta 1862: 461-471, 2016.

3. Miller DH, Chard DT and Ciccarelli O: Clinically isolated syndromes. Lancet Neurol 11: 157-169, 2012.

4. Kappos L, Polman CH, Freedman MS, Edan G, Hartung HP, Miller DH, Montalban X, Barkhof F, Bauer L, Jakobs P, et al: Treatment with interferon beta-1b delays conversion to clinically definite and McDonald MS in patients with clinically isolated syndromes. Neurology 67: 1242-1249, 2006.

5. Fu Y, Talavage TM and Cheng JX: New imaging techniques in the diagnosis of multiple sclerosis. Expert Opin Med Diagn 2: 1055-1065, 2008.

6. Horsfield MA, Rovaris M, Rocca MA, Rossi P, Benedict RH, Filippi $M$ and Bakshi R: Whole-brain atrophy in multiple sclerosis measured by two segmentation processes from various MRI sequences. J Neurol Sci 216: 169-177, 2003.

7. van Walderveen MA, Kamphorst W, Scheltens P, van Waesberghe JH, Ravid R, Valk J, Polman CH and Barkhof F: Histopathologic correlate of hypointense lesions on T1-weighted spin-echo MRI in multiple sclerosis. Neurology 50: 1282-1288, 1998.

8. Bakshi R, Ariyaratana S, Benedict RH and Jacobs L: Fluid-attenuated inversion recovery magnetic resonance imaging detects cortical and juxtacortical multiple sclerosis lesions. Arch Neurol 58: 742-748, 2001

9. Polman CH, Reingold SC, Edan G, Filippi M, Hartung HP, Kappos L, Lublin FD, Metz LM, McFarland HF, O'Connor PW, et al: Diagnostic criteria for multiple sclerosis: 2005 revisions to the 'McDonald Criteria'. Ann Neurol 58: 840-846, 2005

10. Richards TL: Proton MR spectroscopy in multiple sclerosis: Value in establishing diagnosis, monitoring progression, and evaluating therapy. AJR Am J Roentgenol 157: 1073-1078, 1991.

11. Pike GB, De Stefano N, Narayanan S, Worsley KJ, Pelletier D, Francis GS, Antel JP and Arnold DL: Multiple sclerosis: Magnetization transfer MR imaging of white matter before lesion appearance on T2-weighted images. Radiology 215: 824-830, 2000

12. Filippi M and Rocca MA: Magnetization transfer magnetic resonance imaging in the assessment of neurological diseases. J Neuroimaging 14: 303-313, 2004.

13. Rovaris M, Gass A, Bammer R, Hickman SJ, Ciccarelli O, Miller DH and Filippi M: Diffusion MRI in multiple sclerosis. Neurology 65: 1526-1532, 2005

14. Lapointe E, Li DKB, Traboulsee AL and Rauscher A: What Have We Learned from Perfusion MRI in Multiple Sclerosis? AJNR Am J Neuroradiol 39: 994-1000, 2018

15. Kotsiantis SB, Zaharakis I and Pintelas P: Supervised machine learning: A review of classification techniques. Emerging artificial intelligence applications in computer engineering 160 : 3-24, 2007

16. Mortazavi D, Kouzani AZ and Soltanian-Zadeh H: Segmentation of multiple sclerosis lesions in MR images: A review. Neuroradiology 54: 299-320, 2012.

17. Uddin $\mathrm{M}$, Wang $\mathrm{Y}$ and Woodbury-Smith $\mathrm{M}$ : Artificial intelligence for precision medicine in neurodevelopmental disorders. NPJ Digit Med 2: 112, 2019.

18. Bergsland N, Horakova D, Dwyer MG, Uher T, Vaneckova M Tyblova M, Seidl Z, Krasensky J, Havrdova E and Zivadinov R: Gray matter atrophy patterns in multiple sclerosis: A 10-year source-based morphometry study. Neuroimage Clin 17: 444-451, 2017.

19. Pontillo G, Petracca M, Cocozza S and Brunetti A: The Development of Subcortical Gray Matter Atrophy in Multiple Sclerosis: One Size Does Not Fit All. AJNR Am J Neuroradiol 41: E80-E81, 2020.

20. Almutairi AH, Hassan HA, Suppiah S, Alomair OI, Alshoaibi A, Almutairi $\mathrm{H}$ and Mahmud R: Lesion load assessment among multiple sclerosis patient using DIR, FLAIR, and T2WI sequences. Egypt J Radiol Nucl Med 51: 209, 2020.
21. Thompson AJ, Banwell BL, Barkhof F, Carroll WM, Coetzee T, Comi G, Correale J, Fazekas F, Filippi M, Freedman MS, et al: Diagnosis of multiple sclerosis: 2017 revisions of the McDonald criteria. Lancet Neurol 17: 162-173, 2018.

22. Valverde $S$, Cabezas M, Roura E, González-Villà S, Pareto D, Vilanova JC, Ramió-Torrentà L, Rovira À, Oliver A and Lladó X: Improving automated multiple sclerosis lesion segmentation with a cascaded 3D convolutional neural network approach. Neuroimage 155: 159-168, 2017.

23. Birenbaum A and Greenspan H: Multi-view longitudinal CNN for multiple sclerosis lesion segmentation. Eng Appl Artif Intell 65: 111-118, 2017.

24. Carass A, Roy S, Jog A, Cuzzocreo JL, Magrath E, Gherman A, Button J, Nguyen J, Bazin PL, Calabresi PA, et al: Longitudinal multiple sclerosis lesion segmentation data resource. Data Brief 12: 346-350, 2017.

25. Gros C, De Leener B, Badji A, Maranzano J, Eden D, Dupont SM, Talbott J, Zhuoquiong R, Liu Y, Granberg T, et al: Automatic segmentation of the spinal cord and intramedullary multiple sclerosis lesions with convolutional neural networks. Neuroimage 184: 901-915, 2019.

26. Milletari F, Navab $\mathrm{N}$ and Ahmadi SA: V-Net: Fully Convolutional Neural Networks for Volumetric Medical Image Segmentation. In: Proceedings of the 2016 Fourth International Conference on 3D Vision (3DV). IEEE, Stanford, CA, pp565-571, 2016

27. De Leener B, Kadoury S and Cohen-Adad J: Robust, accurate and fast automatic segmentation of the spinal cord. Neuroimage 98: 528-536, 2014

28. Aslani S, Dayan M, Storelli L, Filippi M, Murino V, Rocca MA and Sona D: Multi-branch convolutional neural network for multiple sclerosis lesion segmentation. Neuroimage 196: 1-15, 2019.

29. Sander L, Pezold S, Andermatt S, Amann M, Meier D, Wendebourg MJ, Sinnecker T, Radue EW, Naegelin Y, Granziera C, et al; Alzheimer's Disease Neuroimaging Initiative: Accurate, rapid and reliable, fully automated MRI brainstem segmentation for application in multiple sclerosis and neurodegenerative diseases. Hum Brain Mapp 40: 4091-4104, 2019.

30. Iglesias JE, Van Leemput K, Bhatt P, Casillas C, Dutt S, Schuff N, Truran-Sacrey D, Boxer A and Fischl B; Alzheimer's Disease Neuroimaging Initiative: Bayesian segmentation of brainstem structures in MRI. Neuroimage 113: 184-195, 2015.

31. Hashemi SR, Salehi SSM, Erdogmus D, Prabhu SP, Warfield SK and Gholipour A: Asymmetric Loss Functions and Deep Densely Connected Networks for Highly Imbalanced Medical Image Segmentation: Application to Multiple Sclerosis Lesion Detection. IEEE Access 7: 721-1735, 2019.

32. Commowick O, Cervenansky $\mathrm{F}$ and Ameli R: MSSEG challenge proceedings: multiple sclerosis lesions segmentation challenge using a data management and processing infrastructure. MICCAI, Athens, 2016.

33. Carass A, Roy S, Jog A, Cuzzocreo JL, Magrath E, Gherman A, Button J, Nguyen J, Prados F, Sudre CH, et al: Longitudinal multiple sclerosis lesion segmentation: Resource and challenge. Neuroimage 148: 77-102, 2017.

34. Gabr RE, Coronado I, Robinson M, Sujit SJ, Datta S, Sun X, Allen WJ, Lublin FD, Wolinsky JS and Narayana PA: Brain and lesion segmentation in multiple sclerosis using fully convolutional neural networks: A large-scale study. Mult Scler 26: $1217-1226,2020$

35. Weeda MM, Brouwer I, de Vos ML, de Vries MS, Barkhof F, Pouwels PJW and Vrenken H: Comparing lesion segmentation methods in multiple sclerosis: Input from one manually delineated subject is sufficient for accurate lesion segmentation. Neuroimage Clin 24: 102074, 2019.

36. Valverde S, Salem M, Cabezas M, Pareto D, Vilanova JC, Ramió-Torrentà L, Rovira À, Salvi J, Oliver A and Lladó X: One-shot domain adaptation in multiple sclerosis lesion segmentation using convolutional neural networks. Neuroimage Clin 21: $101638,2019$.

37. Shiee N, Bazin PL, Ozturk A, Reich DS, Calabresi PA and Pham DL: A topology-preserving approach to the segmentation of brain images with multiple sclerosis lesions. Neuroimage 49: 1524-1535, 2010.

38. Schmidt P: Bayesian Inference for Structured Additive Regression Models for Large-Scale Problems with Applications to Medical Imaging (unpublished PhD thesis). Ludwig-MaximiliansUniversität München, 2016 
39. Griffanti L,Zamboni G, Khan A,Li L, Bonifacio G, Sundaresan V, Schulz UG, Kuker W, Battaglini M, Rothwell PM, et al: BIANCA (Brain Intensity AbNormality Classification Algorithm): A new tool for automated segmentation of white matter hyperintensities. Neuroimage 141: 191-205, 2016.

40. McKinley R, Wepfer R, Aschwanden F, Grunder L, Muri R, Rummel C, Verma R, Weisstanner C, Reyes M, Salmen A, et al: Simultaneous lesion and neuroanatomy segmentation in multiple sclerosis using deep neural networks. arXiv:1901.07419.

41. Narayana PA, Coronado I, Sujit SJ, Wolinsky JS, Lublin FD and Gabr RE: Deep-Learning-Based Neural Tissue Segmentation of MRI in Multiple Sclerosis: Effect of Training Set Size. J Magn Reson Imaging 51: 1487-1496, 2020.

42. Nair T, Precup D, Arnold DL and Arbel T: Exploring uncertainty measures in deep networks for Multiple sclerosis lesion detection and segmentation. Med Image Anal 59: 101557, 2020.

43. McKinley R, Wepfer R, Aschwanden F, Grunder L, Muri R, Rummel C, Verma R, Weisstanner C, Reyes M, Salmen A, et al: Simultaneous lesion and brain segmentation in multiple sclerosis using deep neural networks. Sci Rep 11: 1087, 2021.

44. Narayana PA, Coronado I, Sujit SJ, Sun X, Wolinsky JS and Gabr RE: Are multi-contrast magnetic resonance images necessary for segmenting multiple sclerosis brains? A large cohort study based on deep learning. Magn Reson Imaging 65 : $8-14,2020$.

45. Salem M, Valverde S, Cabezas M, Pareto D, Oliver A, Salvi J, Rovira À and Lladó X: A fully convolutional neural network for new T2-w lesion detection in multiple sclerosis. Neuroimage Clin 25: 102149, 2020.

46. Brown RA, Fetco D, Fratila R, Fadda G, Jiang S, Alkhawajah NM, Yeh EA, Banwell B, Bar-Or A and Arnold DL; Canadian Pediatric Demyelinating Disease Network: Deep learning segmentation of orbital fat to calibrate conventional MRI for longitudinal studies. Neuroimage 208: 116442, 2020.

47. Ackaouy A, Courty N, Vallée E, Commowick O, Barillot C and Galassi F: Unsupervised Domain Adaptation With Optimal Transport in Multi-Site Segmentation of Multiple Sclerosis Lesions From MRI Data. Front Comput Neurosci 14: 19, 2020

48. Commowick O, Istace A, Kain M, Laurent B, Leray F, Simon M, Pop SC, Girard P, Améli R, Ferré JC, et al: Objective Evaluation of Multiple Sclerosis Lesion Segmentation using a Data Management and Processing Infrastructure. Sci Rep 8: 13650, 2018.

49. Coronado I, Gabr RE and Narayana PA: Deep learning segmentation of gadolinium-enhancing lesions in multiple sclerosis. Mult Scler 27: 219-527, 2021

50. La Rosa F, Abdulkadir A, Fartaria MJ, Rahmanzadeh R Lu PJ, Galbusera R, Barakovic M, Thiran JP, Granziera C and Cuadra MB: Multiple sclerosis cortical and WM lesion segmentation at 3T MRI: A deep learning method based on FLAIR and MP2RAGE. Neuroimage Clin 27: 102335, 2020.

51. Gessert N, Krüger J, Opfer R, Ostwaldt AC, Manogaran P, Kitzler HH, Schippling S and Schlaefer A: Multiple sclerosis lesion activity segmentation with attention-guided two-path CNNs. Comput Med Imaging Graph 84: 101772, 2020.

52. Essa E, Aldesouky D, Hussein SE and Rashad MZ: Neuro-fuzzy patch-wise R-CNN for multiple sclerosis segmentation. Med Biol Eng Comput 58: 2161-2175, 2020.

53. Barquero G, La Rosa F, Kebiri H, Lu PJ, Rahmanzadeh R, Weigel M, Fartaria MJ, Kober T, Théaudin M, Du Pasquier R, et al: RimNet: A deep 3D multimodal MRI architecture for paramagnetic rim lesion assessment in multiple sclerosis. Neuroimage Clin 28: 102412, 2020.

54. Gautam R and Sharma M: Prevalence and Diagnosis of Neurological Disorders Using Different Deep Learning Techniques: A Meta-Analysis. J Med Syst 44: 49, 2020.

55. Yoo Y, Tang LYW, Brosch T, Li DKB, Kolind S, Vavasour I, Rauscher A, MacKay AL, Traboulsee A and Tam RC: Deep learning of joint myelin and T1w MRI features in normal-appearing brain tissue to distinguish between multiple sclerosis patients and healthy controls. Neuroimage Clin 17: $169-178,2017$

56. Wang SH, Tang C, Sun J, Yang J, Huang C, Phillips P and Zhang YD: Multiple Sclerosis Identification by 14-Layer Convolutional Neural Network With Batch Normalization, Dropout, and Stochastic Pooling. Front Neurosci 12: 818, 2018.

57. Zhang YD, Pan C, Sun J and Tang C: Multiple sclerosis identification by convolutional neural network with dropout and parametric ReLU. J Comput Sci 28: 818, 2018.
58. Talo M, Baloglu UB, Yıldırım Ö and Acharya UR: Application of deep transfer learning for automated brain abnormality classification using MR images. Cogn Syst Res 54: 176-188, 2019.

59. Lu S, Lu Z and Zhang YD: Pathological brain detection based on AlexNet and transfer learning. J Comput Sci 30: 41-47, 2019.

60. McKinley R, Wepfer R, Grunder L, Aschwanden F, Fischer T, Friedli C, Muri R, Rummel C, Verma R, Weisstanner C, et al: Automatic detection of lesion load change in Multiple Sclerosis using convolutional neural networks with segmentation confidence. Neuroimage Clin 25: 102104, 2020.

61. Marzullo A, Kocevar G, Stamile C, Durand-Dubief F, Terracina G, Calimeri F and Sappey-Marinier D: Classification of Multiple Sclerosis Clinical Profiles via Graph Convolutional Neural Networks. Front Neurosci 13: 594, 2019.

62. Eitel F, Soehler E, Bellmann-Strobl J, Brandt AU, Ruprecht K, Giess RM, Kuchling J, Asseyer S, Weygandt M, Haynes JD, et al: Uncovering convolutional neural network decisions for diagnosing multiple sclerosis on conventional MRI using layer-wise relevance propagation. Neuroimage Clin 24: 102003, 2019.

63. Narayana PA, Coronado I, Sujit SJ, Wolinsky JS, Lublin FD and Gabr RE: Deep Learning for Predicting Enhancing Lesions in Multiple Sclerosis from Noncontrast MRI. Radiology 294: 398-404, 2020.

64. Maggi P, Fartaria MJ, Jorge J, La Rosa F, Absinta M, Sati P, Meuli R, Du Pasquier R, Reich DS, Cuadra MB, et al: CVSnet: A machine learning approach for automated central vein sign assessment in multiple sclerosis. NMR Biomed 33: e4283, 2020.

65. Wang Z, Yu Z, Wang Y, Zhang H, Luo Y, Shi L, Wang Y and Guo C: 3D Compressed Convolutional Neural Network Differentiates Neuromyelitis Optical Spectrum Disorders From Multiple Sclerosis Using Automated White Matter Hyperintensities Segmentations. Front Physiol 11: 612928, 2020.

66. Roca P, Attye A, Colas L, Tucholka A, Rubini P, Cackowski S, Ding J, Budzik JF, Renard F, Doyle S, et al; OFSEP Investigators; Steering Committee; Investigators; Imaging group: Artificial intelligence to predict clinical disability in patients with multiple sclerosis using FLAIR MRI. Diagn Interv Imaging 101: 795-802, 2020.

67. Lopatina A, Ropele S, Sibgatulin R, Reichenbach JR and Güllmar D: Investigation of Deep-Learning-Driven Identification of Multiple Sclerosis Patients Based on Susceptibility-Weighted Images Using Relevance Analysis. Front Neurosci 14: 609468, 2020.

68. Sreekumari A, Shanbhag D, Yeo D, Foo T, Pilitsis J, Polzin J, Patil U, Coblentz A, Kapadia A, Khinda J, et al: A Deep Learning-Based Approach to Reduce Rescan and Recall Rates in Clinical MRI Examinations. AJNR Am J Neuroradiol 40: 217-223, 2019.

69. Sujit SJ, Coronado I, Kamali A, Narayana PA and Gabr RE: Automated image quality evaluation of structural brain MRI using an ensemble of deep learning networks. J Magn Reson Imaging 50: 1260-1267, 2019.

70. Lublin FD, Cofield SS, Cutter GR, Conwit R, Narayana PA, Nelson F, Salter AR, Gustafson T and Wolinsky JS; CombiRx Investigators: Randomized study combining interferon and glatiramer acetate in multiple sclerosis. Ann Neurol 73: 327-340, 2013.

71. Zhao C, Shao M, Carass A, Li H, Dewey BE, Ellingsen LM, Woo J, Guttman MA, Blitz AM, Stone M, et al: Applications of a deep learning method for anti-aliasing and super-resolution in MRI. Magn Reson Imaging 64: 132-141, 2019.

72. Jog A, Carass A and Prince JL: Self Super-resolution for Magnetic Resonance Images. Med Image Comput Comput Assist Interv 9902: 553-560, 2016.

73. Wei W, Poirion E, Bodini B, Durrleman S, Colliot O, Stankoff B and Ayache N: Fluid-attenuated inversion recovery MRI synthesis from multisequence MRI using three-dimensional fully convolutional networks for multiple sclerosis. J Med Imaging (Bellingham) 6: 014005, 2019.

74. Ye DH, Zikic D, Glocker B, Criminisi A and Konukoglu E: Modality propagation: coherent synthesis of subject-specific scans with data-driven regularization. Med Image Comput Comput Assist Interv 13: 606-613, 2013.

75. Jog A, Carass A, Pham DL and Prince JL: RANDOM FOREST FLAIR RECONSTRUCTION FROM $T_{1}, T_{2}$ AND $P_{D}$-WEIGHTED MRI. Proc IEEE Int Symp Biomed Imaging 2014: 1079-1082, 2014.

76. Ronneberger O, Fischer P and Brox T: U-Net: Convolutional Networks for Biomedical Image Segmentation. Lect Notes Comput Sci 9351: 234-241, 2015. 
77. Salem M, Valverde S, Cabezas M, Pareto D, Oliver A, Salvi J, Rovira Â and Lladó X: Multiple sclerosis lesion synthesis in MRI using an encoder-decoder U-NET. IEEE Access 7: 25171-25184, 2019.

78. Wei W, Poirion E, Bodini B, Durrleman S, Ayache N, Stankoff B and Colliot O: Predicting PET-derived demyelination from multimodal MRI using sketcher-refiner adversarial training for multiple sclerosis. Med Image Anal 58: 101546, 2019.

79. Finck T, Li H, Grundl L, Eichinger P, Bussas M, Mühlau M, Menze B and Wiestler B: Deep-Learning Generated Synthetic Double Inversion Recovery Images Improve Multiple Sclerosis Lesion Detection. Invest Radiol 55: 318-323, 2020.

80. Yoon J, Gong E, Chatnuntawech I, Bilgic B, Lee J, Jung W, Ko J, Jung H, Setsompop K, Zaharchuk G, et al: Quantitative susceptibility mapping using deep neural network: QSMnet. Neuroimage 179: 199-206, 2018.

81. Bollmann S, Rasmussen KGB, Kristensen M, Blendal RG, Østergaard LR, Plocharski M, O'Brien K, Langkammer C, Janke A and Barth M: DeepQSM-using deep learning to solve the dipole inversion for quantitative susceptibility mapping. Neuroimage 195: 373-383, 2019.
82. Dewey BE, Zhao C, Reinhold JC, Carass A, Fitzgerald KC, Sotirchos ES, Saidha S, Oh J, Pham DL, Calabresi PA, et al: DeepHarmony: A deep learning approach to contrast harmonization across scanner changes. Magn Reson Imaging 64: 160-170, 2019.

83. Liu H, Xiang QS, Tam R, Dvorak AV, MacKay AL, Kolind SH, Traboulsee A, Vavasour IM, Li DKB, Kramer JK, et al: Myelin water imaging data analysis in less than one minute. Neuroimage 210: 116551, 2020.

This work is licensed under a Creative Commons Attribution-NonCommercial-NoDerivatives 4.0 International (CC BY-NC-ND 4.0) License. 